\title{
Application of Sumudo Transform and Homotopy Perturbation Method to Solve Nonlinear Differential Equations
}

\author{
A.D. CHINDHE ${ }^{1}$, S.B. KIWNE ${ }^{2}$ \\ ${ }^{1}$ Balbhim College, Beed, Maharashtra, India \\ 2 Deogiri College, Aurangabad, Maharashtra, India
}

\section{Email: anilchindhe5@gmail.com}

Received: May 24, 2017| Revised: July 15, 2017 I Accepted: August 14, 2017

Published Online: September 01, 2017

The Author(s) 2017. This article is published with open access at www.chitkara.edu.in/publications

Abstract: In this paper the Sumudo transform is coupled with Homotopy Perturbation Method to have the numerical solution of some nonlinear differential equations. The basic concept of applying such technique is to handled the nonlinear terms by using the He's polynomial.

Keywords: Sumudo Transform, Homotopy Perturbation Method, Logistic Differential Equation.

\section{INTRODUCTION}

In the literature survey we can observed the nonlinear phenomenon in many scientific and engineering application which are in the form of ordinary

differential equations and partial differential equations. There are several techniques to solve such nonlinear ordinary differential equations and partial differential equations. Mahmoud S. Rawashdeh and Shehu Maitama [2] have introduced Natural Decomposition Method to solve nonlinear ordinary differential equations. There are other techniques to handle these nonlinear ordinary differential equations and partial differential equations. However such techniques needs large number of numerical computations. This paper deals with the method in which the Sumudo transform and Homotopy Perturbation Method are combined to solve some nonlinear differential equations. The nonlinear terms that occurs in the equations are decomposed using $\mathrm{He}$ 's Polynomials.[1]

Recently Hassan Eltayeb and Adem Kilicman have developed the Sumudo Transform Method to solve the nonlinear system of partial differential equations and Volterra integro-differential equations.[4, 5] Jagdev Singh,

Mathematical Journal of Interdisciplinary Sciences Vol-6, No-1, September 2017 pp. 1-9 
Chindhe, A.D. Devendra Kumar and Adem Kilicman [6] have introduced the combined Kiwne, S.B. Homotopy Perturbation Method and Sumudu Transform to solve nonlinear Fractional Gas Dynamics equations.In this paper the numerical solution of logistic differential equation and Lokta-Volterra predator-prey model for the single species is obtained using the Sumudo Homotopy Perturbation Method(SHPM).

In early 90's Watugala [3] have introduced new integral transform the Sumudo transform which is defined over the set of functions

$$
\left.A=\left[f(t) / \exists \mathrm{M}, \tau_{1}, \tau_{2}>0,|\mathrm{f}(t)|<M e^{\frac{|t|}{\tau_{j}}}\right], \quad \text { if } t \in(-1)^{j} \times[0, \infty)\right] \text { by the }
$$

formula

$$
\mathbb{S}[f(t)]=G(u)=\int_{0}^{\infty} e^{-t} f(u t) d t \quad u \in\left(-\tau_{1}, \tau_{2}\right)
$$

Further details and properties about sumudo tranform can be seen in $[7,8,9,10]$.

\subsection{Some Standard Result of Sumudo Transform}

In this section we assume that all the considered functions are such that their Sumudo tranform exists.[9]

1. $\mathbb{S}[1]=1$

2. $\quad \mathbb{S}[\mathrm{t}]=\mathrm{u}$

3. $\mathbb{S}\left[\frac{t^{n-1}}{(n-1) !}\right]=u^{n-1}$ for $\mathrm{n}=1,2, \ldots$

4. $\quad \mathbb{S}\left[e^{a t}\right]=\frac{1}{1-a u}$

5. $\mathbb{S}\left[\frac{\sin (a t)}{a}\right]=\frac{u}{1+s^{2} u^{2}}$

6. $\quad \mathbb{S}[\cos (a t)]=\frac{1}{1+s^{2} u^{2}}$

7. $\mathbb{S}\left[\frac{t^{n-1} e^{a t}}{(n-1) !}\right]=\frac{u^{n-1}}{(1-a u)^{2}}$

8. $\mathbb{S}[f(n)(t)]=\frac{1}{u^{n}} \cdot G(u)-\sum_{k=0}^{n-1} \frac{f^{k}(0)}{u^{n-k}}$ 


\section{SOLUTION OF NON-LINEAR EQUATIONS (PART-I)}

The aim of this section is to discuss the use of sumudo transform algorithm to solve some nonlinear differential equations. To illustrate the basic technique, consider the following second order non-homogeneous nonlinear differential equation with the given initial condition of the form

$$
D x(t)+R x(t)+N x(t)=g(t)
$$

where $x(0)=A, x^{\prime}(0)=B$ are constants, $D=\frac{d^{2}}{d t^{2}}$ is the second order differential operator, $\mathrm{R}$ is the remaining linear operator, $\mathrm{N}$ is the general nonlinear differential operator and $\mathrm{g}(\mathrm{t})$ is a source term.

First apply the sumudo transform on both sides of the equation (2) we get,

$$
\mathbb{S}[D x(t)]+\mathbb{S}[R x(t)]+\mathbb{S}[N x(t)]=\mathbb{S}[g(t)]
$$

Using the properties of sumudo transform we have,

$$
\mathbb{S}[x(t)]=x(0)-x^{\prime}(0) \cdot u+u^{2} \mathbb{S}[g(t)-N x(t)-R x(t)]
$$

Now apply inverse Sumudo transform on both sides, we get

$$
x(t)=x(0)-x^{\prime}(0) \cdot t+\mathbb{S}^{-1}\left[u^{2} \mathbb{S}[g(t)-N x(t)-R x(t)]\right]
$$

To find the exact solution $\mathrm{x}(\mathrm{t})$ we apply the Homotopy Perturbation Method for which consider $x(t)=\sum_{n=0}^{\infty} P^{n} x_{n}(t)$ and decompose the nonlinear term into the form $N x(t)=\sum_{n=0}^{\infty} P^{n} H_{n}(t)$ where $\mathrm{Hn}(\mathrm{t})$ are the He's polynomial which can be calculated by the formula

$$
H_{n}=\frac{1}{n !} \frac{d^{n}}{d P^{n}}\left(N\left(\sum_{n=0}^{\infty} P^{n} X_{n}\right)\right) n=0,1,2 \ldots
$$

$\therefore$ Equation (5)becomes

$$
\sum_{n=0}^{\infty} P^{n} x_{n}(t)=x(0)-x^{\prime}(0) \cdot t+\mathbb{S}^{-1}\left[\begin{array}{l}
u^{2} \mathbb{S}[g(t)]-p \mathbb{S}^{-1}\left[u^{2} \mathbb{S}\right] \\
{\left[-\sum_{n=0}^{\infty} P^{n} H_{n}(t)-\sum_{n=0}^{\infty} P^{n} x_{n}(t)\right]}
\end{array}\right]
$$

From this last equation we have the recursive relation as
Application of Sumudo Transform and Homotopy

Perturbation Method to Solve Nonlinear Differential Equations 
Chindhe, A.D.

Kiwne, S.B.

$$
\begin{gathered}
x_{0}(t)=H(t)=x(0)-x^{\prime}(0) t+\mathbb{S}^{-1}\left[u^{2} \cdot \mathbb{S}[g(t)]\right] \text { and } \\
x_{n+1}(t)=-\mathbb{S}^{-1}\left[R \sum_{n=0}^{\infty} P^{n} x_{n}(t)+\sum_{n=0}^{\infty} P^{n} H_{n}(t)\right]
\end{gathered}
$$

This gives us the series solution of the given second order non-homogeneous nonlinear differential equation with the given initial condition.

\subsection{Analysis of Method}

Consider the logistic differential equation for the growth of population of single species of the form [12]

$$
\frac{d P}{d t}=r \cdot P\left[1-\frac{P}{k}\right]
$$

Where $\mathrm{r}$ and $\mathrm{k}$ are constants and $\mathrm{P}=\mathrm{P}(\mathrm{t})$ represents the population of species at time $\mathrm{t}$ and $r\left[1-\frac{P}{k}\right]$ is the per capita growth rate, $\mathrm{k}$ is the carrying capacity of the environment. Suppose that

$$
X(r)=\frac{P(t)}{k}, \quad \omega=r t
$$

which gives

$$
\frac{d X}{d \omega}=X(1-X)
$$

with initial condition $X(0)=\frac{P_{0}}{k}$ where $P_{0}=P(0)$.

Apply the sumudo transform on both sides we get

$$
\mathbb{S}\left[\frac{d X}{d \omega}\right]=\mathbb{S}[X(1-X)]
$$

Using the properties of sumudo transform we have

$$
\mathbb{S}[X(\omega)]=\frac{P_{0}}{x}+u \cdot \mathbb{S}\left[X-X^{2}\right]
$$

Apply the inverse sumudo transform on both sides 


$$
X(\omega)=\frac{P_{0}}{k}+\mathbb{S}^{-1}\left[u . \mathbb{S}\left[X-X^{2}\right]\right]
$$

Now applying the classical Homotopy perturbation technique for which consider the solution of given logistic differential equation of the form

$$
X(\omega)=\sum_{n=0}^{\infty} P^{n} X_{n}(\omega)
$$

Application of Sumudo Transform and Homotopy

Perturbation Method to Solve Nonlinear Differential Equations

so that the equation (12) becomes

$$
\sum_{n=0}^{\infty} P^{n} X_{n}(\omega)=\frac{P_{0}}{k}+P . \mathbb{S}^{-1}\left[u . \mathbb{S}\left[\sum_{n=0}^{\infty} P^{n} X_{n}(\omega)-\sum_{n=0}^{\infty} P^{n} H_{n}(\omega)\right]\right]
$$

Where $H_{n}(\omega)$ are the He's polynomial which can be calculated using the formula

$$
H_{n}(X)=\frac{1}{n !} \frac{d^{n}}{d P^{n}}\left(N\left(\sum_{n=0}^{\infty} P^{n} X_{n}\right)\right) n=0,1,2 \ldots
$$

Now equating the terms with identical powers of $\mathrm{P}$, we get

$$
P^{0}: X_{0}(\omega)=\frac{P_{0}}{k}
$$

for the numerical purpose suppose that $\mathrm{P}_{0}=3, k=1$ so that $\frac{P_{0}}{k}=3$

$$
\begin{gathered}
P^{1}: X_{1}(\omega)=\mathbb{S}^{-1}\left[u \cdot \mathbb{S}\left[X_{0}-H_{0}\right]\right]=\left(\frac{P_{0}}{k}-\frac{P_{0}^{2}}{k^{2}}\right) \omega \\
P^{1}: X_{1}(\omega)=-6 \omega \\
P^{2}: X_{2}(\omega)=\mathbb{S}^{-1}\left[u \cdot \mathbb{S}\left[X_{1}-H_{1}\right]\right]=15 \omega^{2} \\
P^{3}: X_{3}(\omega)=\mathbb{S}^{-1}\left[u \cdot \mathbb{S}\left[X_{2}-H_{2}\right]\right]=-37 \omega^{2} \\
P^{4}: X_{4}(\omega)=\mathbb{S}^{-1}\left[u \cdot \mathbb{S}\left[X_{3}-H_{3}\right]\right]=\frac{365}{4} \omega^{4}
\end{gathered}
$$

Continue in this way we get the series solution of the logistic differential equation in the form

$$
\begin{aligned}
X(\omega) & =X_{0}+X_{1}+X_{2}+X_{3}+X_{4}+X_{5}+X_{6} \ldots \\
& =3+(-6 \omega)+15 \omega^{2}+\left(-37 \omega^{3}\right)+\frac{365}{4} \omega^{4} \cdots
\end{aligned}
$$


Chindhe, A.D. Kiwne, S.B.

\section{SOLUTION OF NON-LINEAR EQUATIONS (PART-II)}

Now consider the Lokta-Volterra system which is an integrating species predator-prey model governed by $[11,12,13]$

$$
\begin{aligned}
& \frac{d N}{d t}=N[a-b P] \\
& \frac{d P}{d t}=P[c N-d]
\end{aligned}
$$

where $\mathrm{a}, \mathrm{b}, \mathrm{c}, \mathrm{d}$ are constants $\mathrm{N}=\mathrm{N}(\mathrm{t}), \mathrm{P}=\mathrm{P}(\mathrm{t})$ are the prey predator population at time $t$ respectively.

Suppose that

$$
X(r)=\frac{c}{d} \cdot N(t), Y(r)=\frac{b}{a} \cdot P(t) \quad \omega=r t, \alpha=\frac{d}{a}
$$

So that equation (13), (14) becomes

$$
\begin{gathered}
\frac{d X}{d \omega}=X(1-Y) \\
\frac{d Y}{d \omega}=\alpha \cdot Y(X-1)
\end{gathered}
$$

with initial condition $X(0)=\delta, Y(0)=\beta$

Apply the sumudo transform on both sides we get

$$
\mathbb{S}\left[\frac{d X}{d \omega}\right]=\mathbb{S}[X(1-Y)]
$$

Using the properties of sumudo transform we have

$$
\mathbb{S}[X(\omega)]=\delta+u . \mathbb{S}[X(1-Y)]
$$

Apply the inverse sumudo transform on both sides

$$
X(\omega)=\delta+\mathbb{S}^{-1}[u . \mathbb{S}[X(1-Y)]
$$

Similarly we have

$$
Y(\omega)=\beta+\mathbb{S}^{-1}[u . \alpha . \mathbb{S}[Y(X-1)]]
$$

Let $\delta=1.5, \beta=0.8, \alpha=1$

$$
X(\omega)=1.5+\mathbb{S}^{-1}[u . \mathbb{S}[X(1-Y)]]
$$




$$
X(\omega)=0.8+\mathbb{S}^{-1}[u . \mathbb{S}[Y(X-1)]]
$$

Now applying the classical homotopy perturbation technique for that consider the solution of the form

$$
\begin{gathered}
X(\omega)=\sum_{n=0}^{\infty} P^{n} X_{n}(\omega) \\
Y(\omega)=\sum_{n=0}^{\infty} P^{n} Y_{n}(\omega) \\
\sum_{n=0}^{\infty} p^{n} X_{n}(\omega)=1.5+P . \mathbb{S}^{-1}\left[u . \mathbb{S}\left[\sum_{n=0}^{\infty} P^{n} X_{n}(\omega)-\sum_{n=0}^{\infty} p^{n} H_{n}(\omega)\right]\right] \\
\sum_{n=0}^{\infty} p^{n} Y_{n}(\omega)=0.8+P . \mathbb{S}^{-1}\left[u . \mathbb{S}\left[\sum_{n=0}^{\infty} P^{n} H_{n}(\omega)-\sum_{n=0}^{\infty} p^{n} Y_{n}(\omega)\right]\right]
\end{gathered}
$$

Application of Sumudo Transform and Homotopy

Perturbation Method to Solve Nonlinear Differential Equations

where $H_{n}(\omega)$ are the He's polynomial which can be calculated using the formula

$$
H_{n}(X)=\frac{1}{n !} \frac{d^{n}}{d P^{n}}\left(N\left(\sum_{n=0}^{\infty} P^{n} X_{n}\right)\right) n=0,1,2 \ldots
$$

Now equating the terms with identical powers of $\mathrm{P}$, we get

$$
\begin{aligned}
& P^{0}: X_{0}(\omega)=1.5 \\
& P^{1}: X_{1}(\omega)=\mathbb{S}^{-1}\left[u . \mathbb{S}\left[X_{0}-H_{0}\right]\right] \\
&=0.3 \omega \\
& P^{2}: X_{2}(\omega)=\mathbb{S}^{-1}\left[u . \mathbb{S}\left[X_{1}-H_{1}\right]\right] \\
&=-0.27 \omega^{2} \\
& P^{3}: X_{3}(\omega)=\mathbb{S}^{-1}\left[u . \mathbb{S}\left[X_{2}-H_{2}\right]\right] \\
&=-0.195 \omega^{3} \\
& P^{0}: Y_{0}(\omega)=0.8 \\
& P^{1}: X_{1}(\omega)=\mathbb{S}^{-1}\left[u . \mathbb{S}\left[H_{0}-Y_{0}\right]\right] \\
&=0.4 \omega \\
& P^{2}: Y_{2}(\omega)=\mathbb{S}^{-1}\left[u . \mathbb{S}\left[H_{1}-Y_{1}\right]\right] \\
&=0.22 \omega^{2}
\end{aligned}
$$


Chindhe, A.D.

Kiwne, S.B.

$$
\begin{aligned}
P^{3}: Y_{3}(\omega) & =\mathbb{S}^{-1}\left[u . \mathbb{S}\left[H_{2}-Y_{2}\right]\right] \\
& =0.03166 \omega^{3}
\end{aligned}
$$

continue in this way we get the series solution of the form

$$
\begin{aligned}
X(\omega) & =X_{0}+X_{1}+X_{2}+X_{3}+X_{4}+X_{5}+X_{6} \ldots \\
& =1.5+(0.3 \omega)+\left(-0.27 \omega^{2}\right)+\left(-0.195 \omega^{3}\right) \ldots \\
Y(\omega) & =Y_{0}+Y_{1}+Y_{2}+Y_{3}+Y_{4}+Y_{5}+Y_{6} \ldots \\
& =0.5+(0.4 \omega)+\left(0.22 \omega^{2}\right)+\left(0.03166 \omega^{3}\right)+\ldots
\end{aligned}
$$

\section{CONCLUSION}

This paper provides a new technique for the solution of nonlinear equations. Also we have obtained the series solution of logistic differential equation and Lokta-Volterra system which is an integrating species predator-prey model using this technique. The advantage of this technique is to handle the nonlinear term and reduce the computational work.

\section{REFERENCES}

[1] He J.-H. Homotopy perturbation technique, Computer Methods in Applied Mechanics and Engineering 178(3-4), 257-262, 1999.

[2] Mahmoud S. Rawashdeh and Shehu Maitama Solving nonlinear ordinary differential equations using the NDM, Internat. J. Math. Ed. Sci. Tech. 5(1), 77-88, 2015.

[3] Watugala G. K. Sumudu transform: a new integral transform to solve differential equations and control engineering problems, Model Assisted Statistics and Applications 24(1), 35-43, 1993.

[4] Hassan Eltayeb and Adem Kilicman Application of Sumudu Decomposition Method to Solve Nonlinear System of Partial Differential Equations ,Abstract and Applied Analysis, vol. 2010, Article ID 598702, 11 pages, 2010.

[5] Hassan Eltayeb and Adem Kilicman Application of Sumudu Decomposition Method to Solve Nonlinear System Volterra Integro-differential Equations, Abstract and Applied Analysis Volume 2014, Article ID 503141, 6 pages

[6] Jagdev Singh, Devendra Kumar, and A. KilicmanHomotopy Perturbation Method for Fractional Gas Dynamics Equation Using Sumudu Transform, Abstract and Applied Analysis Volume 2013, Article ID 934060, 8 pages

[7] Asiru M. A. Sumudu transform and solution of integral equations of convolution type, In-ternat. J. Math. Ed. Sci. Tech. 32, 906-910, 2011. 
[8] Belgacem F. B. M., Karaballi A. A. , Kalla L. S. Analytical investigations of the Sumudu transform and applications to integral production equations, Math. Probl. Eng. 3 (4), 103-118, 2003.

[9] Belgacem F. B. M. , Karaballi A. A. Sumudo transform fundamental properties investigations and applications, Journal of Applied Mathematics and Stochastic Analysis 3 (4), 1-23, 2006.

[10] Hassan Eltayeb , Adem Kilicman , Brian Fisher New integral transform and associated distributions, A Integral Transforms Special functions 21, 367-379, 2010 .

[11] Pamuk S. The Decomposition Method For Continuous Population Model : Single and Interacting Species, Appl.Mat Comput. 163, 79-88, 2005.

[12] Murray J.D. Mathematical Biology,springer,berlin , 1993

[13] Baile J.E. and Ollis D.F.Biochemical Engineering Fundamentals, McGrawHill,Inc.Second Edition,Singapore, 1986.
Application of Sumudo Transform and Homotopy

Perturbation Method to Solve Nonlinear Differential Equations 